\title{
Machine learning for nuclear cardiology: The way forward
}

\author{
Sirish Shrestha, MSc, ${ }^{\text {a }}$ and Partho P. Sengupta, MD, $\mathrm{DM}^{\mathrm{a}}$ \\ a WVU Heart \& Vascular Institute, West Virginia University, Morgantown, WV
}

Received Apr 9, 2018; accepted Apr 9, 2018

doi: $10.1007 / \mathrm{s} 12350-018-1284-\mathrm{x}$

\section{See related article, pp. 1746-1754}

Coronary artery disease (CAD) is the single most common cause of death in the developed world-responsible for about 1 in every 5 deaths. ${ }^{1}$ The major challenge in diagnostic and prognosis of the patients with suspected CAD emanates from the associated comorbidities and the heterogeneity of clinical presentations which modifies the performance of commonly used tests like myocardial perfusion single-photon emission computed tomography (SPECT). ${ }^{2}$ This variance in diagnostic performance myocardial perfusion SPECT (MPS) can be attributed to a clinician's difficulty in interpreting the results and extracting information contained in multitude of perfusion and functional parameters. In this regard, machine learning - a subset of artificial intelligence-can leverage the knowledge representation and automated reasoning to detect and extrapolate patterns from the large number of features. To this end, Alonso et al., ${ }^{3}$ in this issue of the Journal of Nuclear Cardiology, illustrate the benefits of machine learning techniques for developing a model to enhance the prognostic value from a complex data of the MPS, electrocardiogram, and clinical variables while increasing interpretability.

Machine learning works in iterations and attempts to learn the data to discern the pattern without much regard for assumptions. The performance of the algorithms improves as the data get larger but sacrifices the interpretability of the features that drives the performance. Machine learning can be trained as a supervised,

Reprint requests: Partho P. Sengupta, MD, DM, WVU Heart \& Vascular Institute, West Virginia University, 1 Medical Center Drive, Morgantown, WV 26506-8059; partho.sengupta@hsc.wvu.edu J Nucl Cardiol 2019;26:1755-8.

$1071-3581 / \$ 34.00$

Copyright (C) 2018 American Society of Nuclear Cardiology. unsupervised, semi-supervised, or reinforcement method to learn from complex disease representation (Table 1); the most frequently studied problems in MPS so far have been supervised. Similarly, Alonso et al. trained an assortment of supervised machine learning algorithms to appraise the accuracy and interpretability in contrast to widely popular logistic regression. In a retrospective analysis, the authors extracted a total of 122 clinical and imaging features from 8321 patients (their database previously reported diverse patient groups including those with valvular heart disease and non-ischemic cardiomyopathy, coronary artery bypass grafting and percutaneous coronary intervention) who underwent dual-isotope MPS with adenosine stress test. All machine learning models were evaluated with fivefold cross-validation for optimization to reduce overfitting. The machine learning models outperformed the parametric statistical model in predicting cardiac death. Support vector machine-a popular classification tool known for its accuracy and generalizability-performed the best among the list of algorithms tested. ${ }^{4}$ Interestingly, however, lasso regression (least absolute shrinkage and selection operator) modified to shrink parameters performed better with only 6 features than the logistic regression with 14 features.

Do the results of the present study mean that our predilection for statistical methods is fading as we become more reliant on machine learning? Perhaps, not categorically; it depends on the purpose of the analysis. There are overlaps in algorithms and methods in the orbits of both statistical models and machine learning algorithms, but the salient characteristic difference must be realized. Machine learning seeks to learn from the data without specific programming, whereas statistical models formalize the relationship between the variables for inference; they both learn from the data, but the purpose and approaches are different. Every statistical model comes with its own set of assumptions that need to be validated using statistical tools ${ }^{5}$ and tactics such as diagnostics, transformation, and model validations to 
Table 1. Types of learning methods ${ }^{6}$

\section{Methods}

1 Supervised

\section{Definition}

Most common learning method applied to the database with labeled outcomes or classes. It involves inferring from labeled training data. Tasks such as regression, classification, predictive modeling, and survival analysis apply supervised learning. Examples include logistic regression, SVM, and Neural Networks

2 Unsupervised A learning method where labeled outcomes or classes do not exist. The goal is to observe similarities and relationships among groups and variables. It is used mostly used in clustering and dimensionality reduction problems. Examples include hierarchical clustering, k-means clustering, and principal component analysis

3 Semi-

A learning method where the data input has a mixture of labeled and unlabeled outcomes or supervised classes. It utilizes the data that are not completely classified. Examples include web-page classification and speech recognition

4 Reinforcement Learning method based on behavioral psychology. The learning agent explores the environment to maximize a reward. It updates based on the feedback it receives from the choices it makes until the 'reward' criteria are met to handle the decision-making function. It is currently being used in medical imaging analytics, disease screening, and personalized prescription selection

determine the quality of the model. Nevertheless, prediction is not a strong suit of statistics since it makes use of estimation of parameters. This was well illustrated in the present investigation where the machine learning classification algorithm - support vector machine (Area under the curve, AUC: 0.83) outperformed parametric regression model-logistic regression (AUC: 0.76).

Several recent studies in Nuclear Cardiology ${ }^{7-13}$ have attempted to use machine learning in the identification of perfusion defects and location, prediction of revascularization and cardiovascular events, and diagnostic and prognostic accuracy (Table 2). However, viewing through the lens of these studies, the investigation by Alonso et al., is singular to apply supervised machine learning in predicting cardiac death from the features while attempting to identify interpretable highperformance model. The investigators additionally demonstrated an enticing visualization tool to risk assessment that is manifested by features' contribution to the risk and patient's ranking with respect to the population. The value of such interpretation scheme would require to be prospectively evaluated in future studies. ${ }^{14}$

While training supervised machine learning model, the feature space derived from the perfusion quantification and clinical variables must be supplied in conjunction with the outcomes. The application of supervised machine learning requires intense preparations in collecting, identifying, and classifying the diseases before the data can be utilized. The outcomes of interests however need to be provided with an inherent assumption that features of interest in the data set are somehow related to the outcome. Unsupervised learning, on the other hand, is example agnostic but finds patterns that are otherwise inconceivable. It can distil high-dimensional data from perfusion and functional parameters to comport naturally occurring patterns in the data. It doesn't require a prior label or annotation of the outcome for the dataset, rather it learns and identifies the relations and uncovers the hidden structure. Therefore, exclusively relying in supervised learning methods in heterogeneous datasets may not produce stellar accuracy, inter-observer variability may be introduced, and bias can creep into the algorithm from semi-quantitatively examined MPS data. However, in conjunction with unsupervised learning, it could ameliorate prediction of the localization and severity of hypo-perfusion that accounts to heterogeneity of the patients and the disease. This may be achieved by condensing and representing the data in a low-dimensional space that is rich in features in identifying severity in hypo-perfusion, or by conglomeration of similar patients or features in clustering, for instance.

Deep learning network is another possibility to extract information from heterogenous data and predict cardiac death with very high accuracy, provided there's enough data for it to train with. ${ }^{15}$ It learns in increment in each hierarchy of the network layers where it extracts predictive features unlike old generations of machine learning algorithms. It performs by learning from examples rather than engineered and hard-coded features. Interestingly, compared to the performance of shallow machine learning algorithms that plateaus for very large dataset, deep learning is scalable and 
Table 2. Application of machine learning in nuclear cardiology

\begin{tabular}{|c|c|c|c|c|c|c|c|}
\hline & Author & $\mathbf{N}$ & Age & Features & Endpoint & $\begin{array}{l}\text { Type of } \\
\text { machine } \\
\text { learning }\end{array}$ & $\begin{array}{c}\text { Overall } \\
\text { accuracy- } \\
\text { AUC }\end{array}$ \\
\hline 1 & $\begin{array}{l}\text { Arsanjani } \\
\text { et al. }\end{array}$ & 713 & $\begin{array}{l}62 \pm 13^{*} \\
66 \pm 11^{\#}\end{array}$ & $\begin{array}{l}\text { Clinical and } \\
\text { quantitative image } \\
\text { features from MPS }\end{array}$ & $\begin{array}{l}\text { Prediction of } \\
\text { revascularization }\end{array}$ & $\begin{array}{l}\text { Ensemble } \\
\text { LogitBoost } \\
\text { classification: } \\
\text { supervised }\end{array}$ & $0.81 \pm 0.02$ \\
\hline 2 & $\begin{array}{l}\text { Arsanjani } \\
\text { et al. }\end{array}$ & 1181 & $\begin{array}{l}64^{\mathrm{b}} \pm 12^{*} \\
48 \pm 11^{\#}\end{array}$ & $\begin{array}{l}\text { Clinical and } \\
\text { quantitative image } \\
\text { features from MPS }\end{array}$ & Prediction of CAD & $\begin{array}{l}\text { Ensemble Logit- } \\
\text { Boost } \\
\text { classification: } \\
\text { supervised }\end{array}$ & $0.81 \pm 0.02$ \\
\hline 3 & $\begin{array}{l}\text { Betancur } \\
\text { et al. }{ }^{9}\end{array}$ & 2619 & $\begin{array}{l}70 \pm 12^{*} \\
62 \pm 11^{\#}\end{array}$ & $\begin{array}{l}\text { Clinical and stress test } \\
\text { results and imaging } \\
\text { features from MPI }\end{array}$ & Prediction of MACE & $\begin{array}{l}\text { Ensemble } \\
\text { LogitBoost } \\
\text { Classification: } \\
\text { supervised }\end{array}$ & $0.81 \pm 0.03$ \\
\hline 4 & $\begin{array}{l}\text { Arsanjani } \\
\text { et al. }^{10}\end{array}$ & 957 & $60 \pm 12$ & $\begin{array}{l}\text { Quantitative perfusion } \\
\text { and functional MPS } \\
\text { variables }\end{array}$ & Prediction of CAD & $\begin{array}{l}\text { Support Vector } \\
\text { Machine } \\
\text { (SVM): } \\
\text { supervised }\end{array}$ & 0.92 \\
\hline 5 & $\begin{array}{l}\text { Nakajima } \\
\text { et al. }{ }^{11}\end{array}$ & 1001 & $69 \pm 10$ & $\begin{array}{l}\text { Clinical and } \\
\text { quantitative data }\end{array}$ & $\begin{array}{l}\text { Diagnostic } \\
\text { accuracy of ANN }\end{array}$ & $\begin{array}{l}\text { Artificial Neural } \\
\text { Network: } \\
\text { supervised }\end{array}$ & 0.91 \\
\hline 6 & $\begin{array}{r}\text { Betancur } \\
\text { et al. }^{12}\end{array}$ & 392 & $62.5 \pm 9.9$ & $\begin{array}{l}\text { Valve Plane position } \\
\text { from intensity, } \\
\text { shape, and } \\
\text { demographic }\end{array}$ & $\begin{array}{l}\text { Automatic valve } \\
\text { plane localization } \\
\text { in MPI }\end{array}$ & SVM: supervised & $0.82 \pm 0.08$ \\
\hline 7 & $\begin{array}{l}\text { Guner } \\
\text { et al. }{ }^{13}\end{array}$ & 308 & $\begin{array}{l}57.5 \pm 10.9^{*} \\
57.9 \pm 12.2^{\#}\end{array}$ & $\begin{array}{l}\text { Clinical and image } \\
\text { from MPS }\end{array}$ & $\begin{array}{l}\text { Detect coronary } \\
\text { artery stenosis }\end{array}$ & $\begin{array}{l}\text { Neural Network: } \\
\text { supervised }\end{array}$ & $\begin{array}{l}0.74^{\mathrm{a}} \\
0.65^{\mathrm{b}} \\
0.72^{\mathrm{c}}\end{array}$ \\
\hline
\end{tabular}

*Group 1

\#Group 2

amages only

bgender and images

'gender, body mass index, and images

continues to perform better with larger data. This capability assisted it to demonstrate its incredible results in recognizing objects in an image, translating languages, driving cars, and remarkable potential in predictive analytics from complex disease representation. Recently, a new deep learning technique called automated Transform by manifold approximation (AUTOMAP) was created which can reconstruct images from range of modalities such as MRI, CT Scan, PET, and X-Ray. It can handle imperfections in the raw data without having the expert to manually tune parameters. ${ }^{16}$ This could translate to lower-dose exposure to patients during perfusion SPECT, or real-time diagnosis while producing high-quality images. It could augment our current diagnosis processes; and whether it be from perfusion SPECT images or a parametric representation of those images such as polar maps, Fourier decomposition, or segmental uptake, deep learning can be a powerful tool to assist clinicians in reconciling data and build patient-specific treatment decisions while improving report accuracy and turnaround time.

Undoubtedly, there will always be challenges and new opportunities, and our first instinct will always be to understand the clinical implications from new ideas. Some of us may question the viability of novel methods while others may embrace it. Some may question the present study for the value of data provided from a retrospective cohort while nuclear cardiology has advanced over the years through novel instrumentation techniques and cameras, and tracers. ${ }^{17}$ While there is 
little doubt that the generalizability of the model would require external verification in a more contemporary data set, it is imperative we recognize that machine learning is more critical to the evolution of nuclear imaging than we ever imagined. And at the frontier of pioneering technologies and rechristening new methods, one of the most important needs is to develop a set of guidelines and standards for the academic world and industry to evaluate ethical, legal and social implications, and ensure interpretability and reproducibility of the machine learning models. ${ }^{18,19}$

\section{Disclosure}

Dr. Sengupta is a consultant for HeartSciences, Hitachi Aloka Ltd., and other authors have nothing to disclose.

\section{References}

1. Cassar A, Holmes DR Jr, Rihal CS, Gersh BJ. Chronic coronary artery disease: Diagnosis and management. Mayo Clin Proc 2009;84:1130-46. https://doi.org/10.4065/mcp.2009.0391.

2. Box LC, Angiolillo DJ, Suzuki N, Box LA, Jian J, Guzman L, et al. Heterogeneity of atherosclerotic plaque characteristics in human coronary artery disease: A three-dimensional intravascular ultrasound study. Catheter Cardiovasc Interv 2007;70(3):349-56. https://doi.org/10.1002/ccd.21088.

3. Alonso DH, Wernick MN, Yang Y, Germano G, Berman DS, Slmoka P. Prediction of cardiac death after adenosine myocardial perfusion SPECT based on machine learning. J Nucl Cardiol 2018. https://doi.org/10.1007/s12350-017-0924-x.

4. Chao CF, Horng MH. The construction of support vector machine classifier using the firefly algorithm. Comput Intell Neurosci 2015. https://doi.org/10.1155/2015/212719.

5. Ivanescu AE, Li P, George B, Brown AW, Keith SW, Raju D, et al. The importance of prediction model validation and assessment in obesity and nutrition research. Int J Obes 2016;40:887-94. https://doi.org/10.1038/ijo.2015.214.

6. Shameer K, Johnson KW, Glicksberg BS, Dudley JT, Sengupta PP. Machine learning in cardiovascular medicine: Are we there yet? Heart 2018. https://doi.org/10.1136/heartjnl-2017-311198.

7. Arsanjani R, Dey D, Khachatryan T, Shalev A, Hayes S, Fish M, et al. Prediction of revascularization after myocardial perfusion
SPECT by machine learning in a large population. J Nucl Cardiol 2015;22:877-84. https://doi.org/10.1007/s12350-014-0027-x.

8. Arsanjani R, Xu Y, Dey D, Vahistha V, Shalev A, Nakanishi R, et al. Improved accuracy of myocardial perfusion SPECT for detection of coronary artery disease by machine learning in a large population. J Nucl Cardiol 2013;20:553-62. https://doi.org/10. 1007/s12350-013-9706-2.

9. Betancur J, Otaki Y, Motwani M, Fish M, Lemley M, Dey D, et al. Prognostic value of combined clinical and myocardial perfusion imaging data using machine learning. J Am Coll Cardiol Imaging 2017. https://doi.org/10.1016/j.jcmg.2017.07.024.

10. Arsanjani R, Xu Y, Dey D, Fish M, Dorbala S, Hayes S, et al. Improved accuracy of myocardial perfusion SPECT for the detection of coronary artery disease by utilizing a support vector machines algorithm. J Nucl Cardiol 2013;54:549-55. https://doi. org/10.2967/jnumed.112.111542.

11. Nakajima K, Kudo T, Nakata T, Kiso K, Kasai T, Taniguchi Y, et al. Diagnostic accuracy of an artificial neural network compared with statistical quantitation of myocardial perfusion images: A Japanese multicenter study. Eur J Nucl Med Mol Imaging 2017;44:2280-9. https://doi.org/10.1007/s00259-017-3834-x.

12. Betancur J, Rubeaux M, Fuchs TA, Otaki Y, Arnson Y, Slipczuk $\mathrm{L}$, et al. Automatic valve plane localization in myocardial perfusion SPECT/CT by machine learning: Anatomic and clinical validation. J Nucl Med 2016;58:961-7. https://doi.org/10.2967/ jnumed.116.179911.

13. Guner LA, Karabacak NI, Akdemir OU, Karagoz PS, Kocaman SA, Cengel A, et al. An open-source framework of neural networks for diagnosis of coronary artery disease from myocardial perfusion SPECT. J Nucl Cardiol 2010;17:405-13. https://doi.org/ 10.1007/s12350-010-9207-5.

14. Intrator $\mathrm{O}$, Intrator $\mathrm{N}$. Interpreting neural-network results: A simulation study. Comput Stat Data Anal 1999;37:373-93. https:// doi.org/10.1016/S0167-9473(01)00016-0.

15. Chen X, Lin X. Big data deep learning: Challenges and perspectives. IEEE Access 2014;2:514-25. https://doi.org/10.1109/access. 2014.2325029.

16. Zhu B, Liu JZ, Cauley SF, Rosen BR, Rosen MS. Image reconstruction by domain-transform manifold learning. Nature 2018;555:487-92. https://doi.org/10.1038/nature25988.

17. De Lorenzo A. The evolving roles of nuclear cardiology. Curr Cardiol Rev 2009;5:52-5. https://doi.org/10.2174/157340309787048112.

18. Wilkinson MD, Dumontier M, Aalbersberg IJ, Appleton G, Axton $\mathrm{M}$, Baak A, et al. The FAIR guiding principles for scientific data management and stewardship. Sci Data 2016;3:160018. https:// doi.org/10.1038/2data.2016.18.

19. Petrick N, Shahiner B, Armato SG, Bert A, Correale L, Delsanto S. Evaluation of computer-aided detection and diagnosis systems. Med Phys 2013;40:087001. https://doi.org/10.1118/1.4816310. 